\title{
Spontaneous pneumomediastinum and pneumothorax in a non-intubated patient with coronavirus disease 2019 pneumonia: A case report
}

\author{
Angela Iuorio, Giacomo Torretta, Pierluigi Fusco, Maurizio Ferrara \\ From Anesthesiologist, Department of Anesthesia and Intensive Care Medicine, Frangipane Hospital, Ariano Irpino, Italy
}

\begin{abstract}
Coronavirus disease 2019 (COVID-19) has rapidly spread across the globe. Many studies have shown its typical and atypical clinical presentations. Pneumothorax and pneumomediastinum have both been noted to complicate cases of COVID-19 requiring hospital admission even in the absence of mechanical ventilation. The major described pathophysiological mechanism is diffuse alveolar injury leading to alveolar rupture and air leak. Here, we describe a case of spontaneous pneumomediastinum and pneumothorax in a nonintubated patient after sixteen days of initial symptoms of COVID-19.
\end{abstract}

Key words: Coronavirus disease 2019, Spontaneous pneumomediastinum, Spontaneous pneumothorax

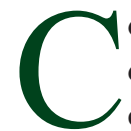
oronavirus disease 2019 (COVID-19) is an infectious disease caused by severe acute respiratory syndrome virus coronavirus 2 (SARS-COV-2). The disease has rapidly spread across the world [1]. It can be diagnosed with real-timereverse transcription-polymerase chain reaction (RT-PCR) from a nasopharyngeal swab and computerized tomography (CT) scan. The common CT manifestations of COVID-19 include a bilateral extensive ground-glass opaque appearance with peripheral or posterior distribution, mainly including the lower lobs. Uncommon features include pleural effusion, lymphadenopathy, cavitation, pneumothorax, and pneumomediastinum [2].

Here, we describe a case of spontaneous pneumomediastinum and pneumothorax in a non-intubated patient after 16 days of initial symptoms of COVID-19. The aim of this study is to describe an increasing complication in COVID-19 patients without the use of invasive or non-invasive positive pressure ventilation and to monitor it as a potential indicator of worsening disease.

\section{CASE REPORT}

An 84-year-old male with a 3 years medical history of hypertension and on drug treatment with angiotensin-converting enzyme inhibitor was admitted to the emergency department with complaints of 6 days of shortness of breath, fever, and persistent cough.

On physical examination, the temperature of the patient was $38^{\circ} \mathrm{C}$, heart rate and respiratory rate were 109 beats/min and

\section{Access this article online}

Received - 23 March 2021

Initial Review - 08 April 2021

Accepted - 08 May 2021

DOI: 10.32677/IJCR.2021.v07.i05.010
$25 / \mathrm{min}$, respectively. The blood pressure was $100 / 60 \mathrm{mmHg}$. The initial peripheral oxygen saturation was $83 \%$ without oxygen and 91\% on non-rebreather mask (NRB) $15 \mathrm{~L} / \mathrm{min}$.

Blood gas analysis revealed $\mathrm{pH}$ of $7.42, \mathrm{pCO}_{2}$ of $30, \mathrm{pO}_{2}$ of $68, \mathrm{HCO} 3$ of 21.7, and lactate of 1.5. Complete blood count and biochemical analysis were obtained. In view of these clinical symptoms, COVID-19 was suspected and real-time RT-PCR was performed from the nasopharyngeal swab. He was found to have severe acute respiratory distress syndrome (ARDS) secondary to SARS-CoV-2 viral pneumonia, with a $\mathrm{PaO} 2 / \mathrm{FiO} 2$ ratio of 110 . Due to the elderly age of the patient, a chest CT was obtained. The CT scan revealed widespread bilateral ground-glass opacities, predominantly in the lower lobes.

He was treated with azithromycin $500 \mathrm{mg} /$ day, dexamethasone $6 \mathrm{mg} / \mathrm{kg} /$ day, low-molecular-weight heparin $4000 \mathrm{UI} /$ day, and remdesivir $200 \mathrm{mg}$ on the $1^{\text {st }}$ day and $100 \mathrm{mg}$ over the next 5 days. He continued to require a $15 \mathrm{~L} / \mathrm{min}$ NRB mask to maintain his oxygenation.

On day 10 of his hospitalization, he developed sudden chest pain, violent coughing, and worsening hypoxemia. He repeated a CT scan that showed (a) increase in bilateral interstitium alveolar thickening areas with a ground-glass and crazy paving appearance and pleural thickening in the posterior region bilaterally, (b) the presence of pneumomediastinum located mainly in the left anterior paracardiac area and along the left hilum (max thickness $15 \mathrm{~mm}$ ), and (c) thin flap of pneumothorax on the left in anteromedial arrangement with a maximum thickness of $8 \mathrm{~mm}$ (Fig. 1). According to a scoring method published in the literature, the total lung severity score

Correspondence to: Dr. Angela Iuorio, Department of Anesthesia and Intensive Care Medicine, Frangipane Hospital, Ariano Irpino, Italy. E-mail: angela.iuorio@ libero.it

(C) 2021 Creative Commons Attribution-NonCommercial 4.0 International License (CC BY-NC-ND 4.0). 


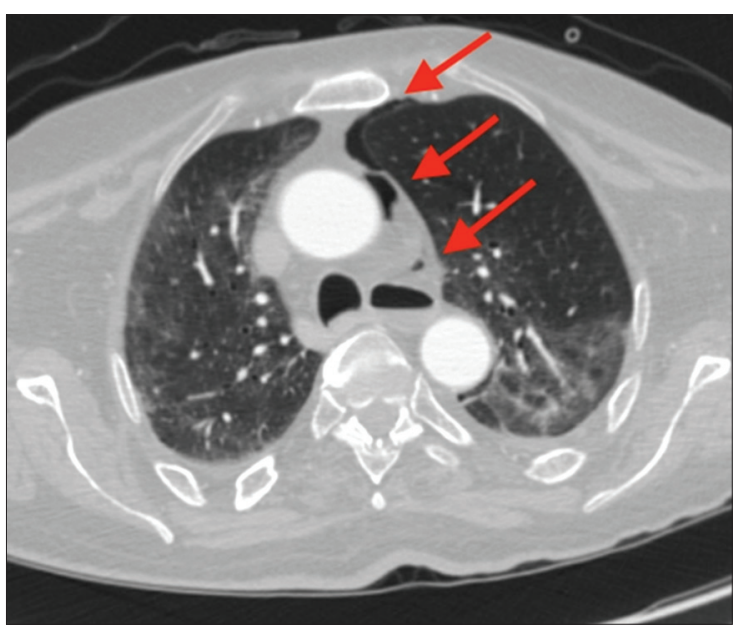

Figure 1: Presence of pneumomediastinum located mainly in the left anterior paracardiac area and along the left hilum (max thickness $15 \mathrm{~mm}$ ). Thin flap of pneumothorax on the left in anteromedial arrangement with a maximum thickness of $8 \mathrm{~mm}$

of the patient was 19 out of 20 [3]. The patient was transferred to ICU for monitored care and after 11 days, died from infectious complication unrelated to Pseudomonas aeruginosa pneumonia.

\section{DISCUSSION}

Spontaneous pneumomediastinum is a rare clinical condition defined as the presence of free air in the mediastinal structures in the absence of a traumatic event or an iatrogenic cause as endotracheal intubation or mechanical ventilation. The main causes of spontaneous pneumomediastinum are asthma, chronic lung disease, corticosteroids, inhaled drug use, some predisposing alterations such as tracheomalacia, and respiratory infections such as pneumocystis pneumonia, Staphylococcus aureus pneumonia, cytomegalovirus, influenza bronchiolitis, and viral pneumonia, including H1N1 influenza [4,5].

In most cases, it is a self-limiting disease, but it can potentially cause severe circulatory and respiratory pathology. The occurrence of spontaneous pneumomediastinum in patients with COVID19 pneumonia is rarely reported and should be monitored as a potential indicator of worsening disease [6].

Spontaneous pneumothorax is a clinical condition defined as the presence of air between the visceral and parietal pleura, which can reduce oxygenation and ventilation. It can be classified as spontaneous (primary and secondary), traumatic, and iatrogenic. In COVID-19 patients, the mechanism of the injury may be secondary to alveolar damage from the infection with rupture of the alveolar wall due to increased pressure from coughing [7]. As per the case report of Tucker et al., SARSCoV-2 infection determines an ARDS leading to the structural changes in the lungs with diffused alveoli wall rupture and interstitial emphysema [8].

Pneumomediastinum and pneumothorax are relatively common complications in patients undergoing mechanical ventilation: The mechanism is barotrauma, which is a trauma caused by increased transalveolar pressure leading to alveoli rupture [9]. The patient described in our case report had only received oxygen therapy through nasal prongs or face mask and did not have a history of smoking or clear parenchymal patterns that suggest bullae or emphysema that could be predisposing factors for the development of pneumothorax.

$\mathrm{He}$ had intense repetitive episodes of cough: These episodes of cough can produce a sudden increase in distal airway pressure, determining alveolar rupture, and secondary gas leakage to the peribronchovascular pulmonary interstitium from where the air can dissect proximally, reaching the mediastinum. This phenomenon is called the "Macklin effect" [10].

El-Hakim et al. described two different pathophysiological mechanisms that can occur simultaneously: One mechanism is an increase in intra-alveolar pressure and overinflation of the alveoli; this pressure gradient can rupture the marginal alveoli, leading to air leaking to the interstitium until it reaches the mediastinum. The other mechanism is caused by a reduction in the caliber of the pulmonary vessels, without a diminution of the alveolar pressure that will increase the pressure gradient causing air leak to the sheath [11].

The clinical evolution of these patients should be monitored for the possibility of pneumomediastinum-pneumothorax-related cardiovascular and respiratory complications.

\section{CONCLUSION}

This case report describes our experience but numerous other data need to be collected. If the presence of spontaneous pneumomediastinum and spontaneous pneumothorax will be identified as an element of disease progression, specific therapeutic recommendations must be decided.

\section{REFERENCES}

1. World Health Organization. Coronavirus Disease (COVID-19) Pandemic. Geneva: World Health Organization; 2020. Available from: https://www. who.int. [Last accessed on $2020 \mathrm{Apr} 10]$.

2. Salehi S, Abedi A, Balakrishnan S, Gholamrezanezhad A. Coronavirus disease 2019 (COVID 19): A systematic review of imaging findings in 919 patients. AJR Am J Roentgenol 2020;14:87-93.

3. Chung M, Bernheim A, Mei X, Zhang N, Huang M, Zeng X, et al. CT imaging features of 2019 novel Coronavirus (2019-nCoV). Radiology 2020;295:202-7.

4. Singh BP, Shetty GS, Vijayan PA, Gopalakrishna U, Chandan G, Santini A, et al. Management of pneumome-diastinum associated with H1N1 pneumonia: A case report. J Crit Care Med (Targu Mures) 2019;5:28-33.

5. Olliff JF, Williams MP. Radiological appearances of cytomegalovirus infections. Clin Radiol 1989;40:463-7.

6. Zhou C, Gao C, Xie Y, Xu M. COVID-19 with spontaneous pneumomediastinum. Lancet Infect Dis 2020;20:510.

7. Sun R, Liu H, Wang X. Mediastinal emphysema, giant bulla, and pneumothorax developed during the course of COVID-19 pneumonia. Korean J Radiol 2020;21:541-4.

8. Tucker L, Patel S, Vatsis C, Poma A, Ammar A, Nasser W, et al. Pneumothorax and pneumomediastinum secondary to COVID-19 disease unrelated to 
mechanical ventilation. Case Rep Crit Care 2020;2020:6655428.

9. Woodside KJ, van Sonnenberg E, Chon KS, Loran DB, Tocino IM, Zwischenberger JB. Pneumothorax in patients with acute respiratory distress syndrome: Pathophysiology, detection, and treatment. J Intensive Care Med 2003;18:9-20.

10. Chassagnon G, Favelle O, Derogis V, Cottier JP. Spontaneous pneumomediastinum due to the Macklin effect: Less is more. Intern Emerg Med 2015;10:759-61.

11. El-Hakim TS, Haalem SA, Romero CP, Rodriguez-Fuentes Y. Spontaneous pneumomediastinum, pneumothorax and subcutaneous emphysema in
COVID-19 pneumonia: A rare case and literature review. BMJ Case Rep 2020;13:e239489.

Funding: None; Conflicts of Interest: None Stated.

How to cite this article: Iuorio A, Torretta G, Fusco P, Ferrara M. Spontaneous pneumomediastinum and pneumothorax in a nonintubated patient with coronavirus disease 2019 pneumonia: A case report. Indian J Child Reports. 2021;7(5):204-206. 